\title{
The PCQP Score for Volume Status of Acutely III Patients: Integrating Vascular Pedicle Width, Caval Index, Respiratory Variability of the QRS Complex and R Wave Amplitude
}

\author{
Ali Taghizadieh, Kavous Shahsavari Nia', Payman Moharramzadeh², Mahboob Pouraghaei², Atefeh Ghavidel'2 Zahra Parsian², Ata Mahmoodpoor ${ }^{3}$ \\ Tuberculosis and Lung Research Center, Tabriz University of Medical Sciences, ${ }^{1}$ Road Traffic Injury Research Center, Tabriz University of Medical Sciences, \\ 2Emergency Medicine Research Team, Tabriz University of Medical Sciences, ${ }^{3}$ Department of Anesthesiology, Faculty of Medicine, Tabriz University of Medical \\ Sciences, Tabriz, Iran
}

\section{Abstract}

Introduction: Techniques for measuring volume status of critically ill patients include invasive, less invasive, or noninvasive ones. The present study aims to assess the accuracy of noninvasive techniques for measuring volume status of critically ill patients. Patients and Methods: A total of 111 critically ill patients admitted to the emergency department and undergoing central venous catheterization were included in the study. Five parameters were measured including vascular pedicle width (VPW), diameter of inferior vena cava, caval index, respiratory changes in QRS, and P wave amplitude. Patients with risk factors which could decrease the accuracy of central venous pressure (CVP) value were excluded from study. We compared these parameters with static CVP parameter. Finally, based on the afore-mentioned parameters, PCQP role in criteria was designed. Results: In detecting loss of circulating blood volume, area under the curve of VPW was 0.92 (90\%, confidence interval [CI]: 0.85-0.99), diameter of inferior vena cava was 0.82 (90\%, CI: $0.72-0.91)$, caval index was 0.9 (90\%, CI: 0.82-0.98), and changes in QRS and P waves were 0.88 (95\%, CI: 0.81-0.95) and 0.73 (95\%, CI: $0.63-$ 0.82), respectively. PCQP role in criteria was designed according to these parameters, and at its best cutoff point (score 6), VPW had a sensitivity of $97.4 \%$ (95\%, CI: 84.57-99.99) and specificity of 83.6\% (95\%, CI: 72.65-90.86) for the detection of loss of circulating blood volume $\left(<8 \mathrm{cmH}_{2} \mathrm{O}\right)$. Conclusion: PCQP score could be a reliable and noninvasive technique for the assessment of volume status in critically ill patients.

Keywords: Caval index, central venous pressure, changes in electrocardiographic wave, diameter of inferior vena cava, pulmonary vein pedicle

\section{INTRODUCTION}

The successful management of critically ill patients in the emergent department is highly dependent on the correct assessment and optimization of volume status. Every emergency department (ED) physician should know and be able to perform an appropriate method to evaluate volume status of patients and manage based on his assessment. However, it is unfortunately very difficult and there are so many ways with different accuracies for the evaluation of volume status in critically ill patients. ${ }^{[1,2]}$ Recent studies greatly emphasize the stabilization of hemodynamic status since it dramatically reduces mortality rate of critically ill patients. ${ }^{[3]}$ Patients with unstable hemodynamic status suffer from greater

\begin{tabular}{|l|l|}
\hline \multicolumn{3}{c|}{ Access this article online } \\
\hline Quick Response Code: & Website: \\
& www.ijccm.org \\
& \\
&
\end{tabular}

complications as well as prolonged hospital stay and greater need for critical care. ${ }^{[4,5]}$

Reliable techniques for measuring circulating blood volume used today are often invasive, and in addition to potential risks and high costs, they are time-consuming and may delay early intervention. Noninvasive diagnostic criteria and tests are less risky and less costly, and they

Address for correspondence: Prof. Ata Mahmoodpoor, General ICU, Shohada Hospital, El-Goli Street, Tabriz, Iran. E-mail: amahmoodpoor@yahoo.com

This is an open access article distributed under the terms of the Creative Commons Attribution-NonCommercial-ShareAlike 3.0 License, which allows others to remix, tweak, and build upon the work non-commercially, as long as the author is credited and the new creations are licensed under the identical terms.

For reprints contact: reprints@medknow.com

How to cite this article: Taghizadieh A, Nia KS, Moharramzadeh $\mathrm{P}$, Pouraghaei M, Ghavidel A, Parsian Z, et al. The PCQP score for volume status of acutely ill patients: Integrating vascular pedicle width, caval index, respiratory variability of the qrs complex and $\mathrm{R}$ wave amplitude. Indian $\mathrm{J}$ Crit Care Med 2017;21:726-32. 
can be performed outside hospital and EDs, but sometimes with lower accuracy compared to invasive ones. ${ }^{[6]}$ One of these methods is vascular pedicle width (VPW) proposed by Milne et al. in 1984 and also in later studies. ${ }^{[7-9]} \mathrm{VPW}$ measurement is relatively simple, accurate, and objective. However, this measurement can be confounded by many factors, including height, physical structure, and technical problems such as patient rotation, low respiratory effort, and supine or standing position of the patient during radiography, underlying diseases, bleeding, and aorta conditions. ${ }^{[10]}$ Diameter of the inferior vena cava is another criterion that can be used to determine intravascular volume and venous return. ${ }^{[11]}$ Many authors believe that dynamics of inferior vena cava dimensions are associated with hemodynamic status. However, this association is not fully understood, and the current knowledge in this area is rather based on qualitative studies. ${ }^{[12,13]}$ Electrocardiographic (ECG) evaluation can also provide a noninvasive diagnostic test for the assessment of intravascular volume status. Studies indicate that morphological changes in ECG may reflect intravascular volume change. For example, a study conducted in 2012 showed that changes in ECG amplitude can be considered an appropriate parameter in determining intravascular volume. ${ }^{[14]}$

Given the above-mentioned, each of these methods, including inferior vena cava changes, VPW, and respiratory changes in ECG, has advantages and disadvantages in assessing circulating blood volume. The present study mainly aims to compare a systematic approach using noninvasive tools, such as chest radiography, ultrasound, and ECG, used to determine intravascular volume with central venous pressure (CVP) values, to enable presentation of a reliable noninvasive criterion for determining status of intravascular volume in critically ill patients admitted to the ED.

\section{Patients and Methods}

\section{Study design and setting}

In this cross-sectional study, participants were critically ill patients admitted to the ED of a university-affiliated hospital. Protocol of the present study was approved by the Ethics Committee of the University. In the course of study, researchers adhered to the Principles of Helsinki Convention. Informed consent forms were signed by the patients at entry to the study.

\section{Patients}

Patients older than 18 years of age and patients who clinically required insertion of central vein catheterization or dialysis catheterization were included. Exclusion criteria included patients with known cardiac anatomy and function disorders, cardiac shunts, intubated patients, pericardial effusion, and pregnancy. Other exclusion criteria were patients with a history of open heart surgery, advanced pulmonary hypertension, hypertrophic cardiomyopathy, and obesity with body mass index $(\mathrm{BMI})>30$. Indication for catheterization was determined by the physicians not involved in this study based on medical needs. Consecutive sampling method was used in this study. Sample size was determined as a minimum of 110 patients based on previous studies ${ }^{[15]}$ and considering $\alpha=0.05$ and $\beta=0.1$.

\section{Measurements}

In this study, six main parameters were assessed.

\section{Central vein pressure}

An emergency specialist determined CVP through central vein catheterization. The patient was positioned in Trendelenburg position $\left(15^{\circ}\right)$, and the catheterization site was sterilized using chlorhexidine. An appropriate catheter was inserted into the right jugular vein with ultrasonography assistance. Following heparinization and suturing of catheter, chest radiograph was taken to ensure correct position of catheter tip. Then, using a ruler, CVP was measured based on midaxillary line and was reported in centimeters of water. ${ }^{[8]}$

\section{Vascular pedicle width}

This was measured according to the anteroposterior chest radiograph in the prone position and radiologist's report (Shimadzu model R-20j 500 MA, made in Japan). Standard focal distance film of 40 inches $(101 \mathrm{~cm})$ was equally applied to all patients, with $\mathrm{Kvp}=90$ and $\mathrm{As}=1 \mathrm{~m}$. Images were recorded on 14 inch $\times 17$ inch films. Measurement on chest radiograph was as follows: A direct line (along body height) was drawn from the point of the left subclavian artery branches off aortic arch, and another line was perpendicularly drawn from that point toward intersection of superior vena cava and the right bronchial vein, and this distance was reported in millimeters. ${ }^{[7]}$

\section{Inferior vena cava diameter}

This diameter was measured using Toshiba Ultrasound Nemio (SSA-550A, Japan). All ultrasounds were performed by a trained emergency medicine specialist and approved by a radiologist. Ultrasounds were evaluated according to a standard protocol, including B-mode images of the inferior vena cava in long and short axes. Transabdominal ultrasound was performed using a $3.5 \mathrm{MHz}$ Curve Transducer (Toshiba, PSF-37HT-3-75MHz, Japan), and images recorded were used to measure diameter of the vein, which included long and short axes images and also a 10-s video recording for the assessment of pulmonary changes of this vein in a complete slow breathing cycle. Orientation of ultrasound transducer in relation to true horizontal was achieved by a leveling device attached to standard ultrasound probe. ${ }^{[16]}$

\section{Caval index}

This criterion is used to assess respiratory changes in vena cava. Caval index was measured by an emergency medicine specialist using ultrasound of inferior vena cava during inhalation and exhalation. The 10-s video recording was used for this purpose. Caval index was calculated using the following equation: 
Cavalindex $\%=$

(inferior vena cava diameter at end of exhalation this diameter at end of inhalation) $\times 100$

(inferior vena cava diameter at end of exhalation)

Amplitude changes in QRS wave

Following ECG and long lead II, this parameter was calculated using equation below and was expressed and recorded in percentage.

QRS amplitude changes $=$

(Highest QRS amplitude in a rspiratory cycle -

$\frac{\text { shortest QRS amplitude in the same cycle) }}{\text { mean of these amplitudes in the same cycle }} \times 100$

\section{Amplitude changes in $P$ wave}

Following ECG and long lead II, this parameter was calculated using equation below.

\section{P amplitude changes $=$}

(Highest $\mathrm{P}$ wave amplitude in a aspiratory cycle -

$\frac{\text { shortest } P \text { wave amplitude in the same cycle) }}{\text { mean of these amplitudes in the same cycle }} \times 100$

\section{Statistical analyses}

Of the 115 patients studied, only $4(3.5 \%)$ had high CVP. Since in terms of the clinical rationale for critically ill patients, low CVP is more important than high CVP, and since few patients in the present study had high CVP, it was decided to divide patients into two groups of patients with low CVP and patients with normal CVP. Accordingly, four patients with high CVP were excluded and only data from 111 patients were analyzed. CVP was considered as the gold standard in the present study. Pressure $<8 \mathrm{~cm}$ of water was regarded as a loss of volume in circulation, between 8 and $15 \mathrm{~cm}$ as normal, and $\geq 16 \mathrm{~cm}$ of water as increased volume in circulation. Data were analyzed using STATA-11.0 software (Stata Corp, College Station, Texas, USA). After ensuring normal distribution of data, relationships of CVP with VPW, inferior vena cava diameter, caval index, and ECG changes were analyzed. Independent $t$-test was used to analyze relationship of these parameters between two groups of patients (with normal CVP and with low CVP). Then, receiver operating characteristic (ROC) curve and area under the curve were calculated for each variable. Sensitivity, specificity, positive and negative likelihood ratios of different parameters were calculated. Finally, a stepwise multivariate logistic regression model was fit to evaluate weight of each parameter in estimation of CVP. To enhance diagnostic values of the above noninvasive tests, a role in model was designed for diagnosing loss of circulating blood volume. In this model, CVP was divided into normal and low groups. Based on regression coefficient for each variable, a weight was assigned and PCQP role in model was designed. In all analyses, $P<0.05$ was considered statistically significant.

\section{RESULTS}

Demographic and basic details of patients

Of 111 participants, $64(55.6 \%)$ were male and $51(44.4 \%)$ were female. Mean age of patients was $66.4 \pm 15.1$ years (ranging from 24 to 95 years), and mean BMI was $24.2 \pm 2.7 \mathrm{~kg} / \mathrm{m}^{2}$ (ranging from 17.5 to 29.5 ). In the present study, five patients $(4.4 \%)$ had low weight, $66(57.4 \%)$ were in the normal range, and $44(38.3 \%)$ were overweight. Mean CVP in patients was $9.5 \pm 3.6 \mathrm{~cm}$ of water (ranging from 2 to $16 \mathrm{~cm}$ of water). Thirty-eight patients $(33 \%)$ had low CVP, and 73 patients $(63.5 \%)$ had normal CVP.

\section{Relationship between factors predicting circulating blood volume and central venous pressure}

Mean VPW, inferior vena cava diameter, changes in inferior vena cava diameter, changes in ECG, and their relationship with CVP are shown in Table 1. It can be seen that there is a direct relationship between VPW, inferior vena cava diameter, and amplitude changes in $\mathrm{P}$ wave and CVP, while caval index and amplitude changes in QRS reduce with increasing volume in circulation.

Value of predicting factors of circulating blood volume in diagnosing circulating blood volume status

The area under venous VPW curve was $0.92(90 \%$, confidence interval [CI]: 0.85-0.99) in diagnosing volume loss [Figure 1]. At the best cutoff point $(\leq 70 \mathrm{~mm})$, VPW had $94.7 \%$ sensitivity and $89 \%$ specificity in diagnosing low CVP. Inferior vena cava diameter was another factor that showed good diagnostic accuracy in this regard. Area under the inferior vena cava diameter curve was 0.82 (95\%, CI: 0.72-0.91) in diagnosing loss of CVP [Figure 1b]. At the best cutoff point $(10.2 \mathrm{~mm})$, this parameter had $84.9 \%$ sensitivity and $71 \%$ specificity. Area under the curve of caval index was 0.9 (90\%, CI: 0.82-0.98) [Figure 1c]. The best cutoff point for caval index was $34.7 \%$, with $89.5 \%$ sensitivity and $72.6 \%$ specificity [Table 2].

Area under the curve of QRS amplitude change was found 0.88 (95\%, CI: 0.81-0.95) [Figure 1d]. The best cutoff point for QRS complex changes was $\geq 20 \%$, with $86.8 \%$ sensitivity and $76.7 \%$ specificity in diagnosing CVP drop. In assessing diagnostic value of changes in $\mathrm{P}$ wave, area under the curve of respiratory $\mathrm{P}$ wave changes was found 0.73 (95\%, CI:

\begin{tabular}{lccc}
\hline $\begin{array}{l}\text { Table 1: Relationship between factors predicting } \\
\text { circulating blood volume and central vein pressure }\end{array}$ \\
\begin{tabular}{lccc} 
Variable & Mean \pm SD & $\boldsymbol{r}$ & $\boldsymbol{P}$ \\
\hline VPW (mm) & $76.9 \pm 14.5$ & 0.54 & $<0.001$ \\
Diameter of IVC (mm) & $4.9 \pm 13.0$ & 0.42 & $<0.001$ \\
Caval index (\%) & $37.3 \pm 13.4$ & -0.58 & $<0.001$ \\
Changes in amplitude of QRS complex (\%) & $21.4 \pm 13.7$ & -0.57 & $<0.001$ \\
Changes in amplitude of P wave (\%) & $16.9 \pm 16.4$ & 0.54 & $<0.001$ \\
\hline
\end{tabular}
\end{tabular}

VPW: Vascular pedicle width; SD: Standard deviation; $r$ : Pearson coefficient; IVC: Inferior vena cava 

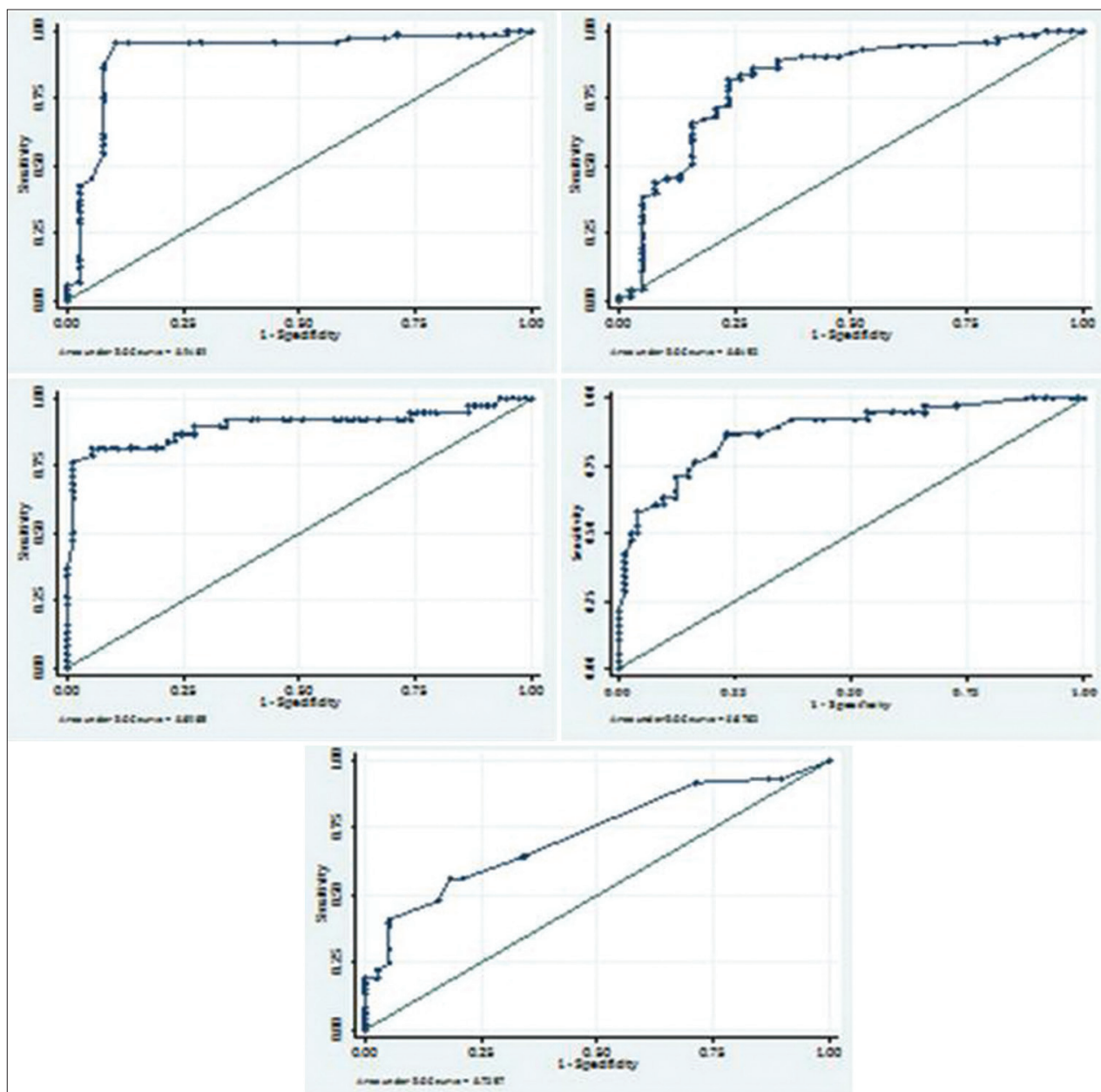

Figure 1: Area under vascular pedicle width receiver operating characteristic curve (a), vena cava diameter (b), caval index (c), QRS complex respiratory changes (d), and changes in respiratory P wave (e)

\section{Table 2: Value of circulating volume predicting factors in diagnosing circulating volume status}

\begin{tabular}{|c|c|c|c|c|c|c|}
\hline Variable & TP & TN & FP & FN & Sensitivity & Specificity \\
\hline Vascular pedicle & 35 & 65 & 8 & 2 & $94.7(80.9-99.1)$ & $89.0(79.0-94.8)$ \\
\hline IVC diameter & 62 & 27 & 11 & 11 & $84.9(74.2-81.9)$ & $71.0(53.9-84.2)$ \\
\hline Caval index & 34 & 53 & 20 & 4 & $89.5(74.3-96.6)$ & $72.6(60.7-82.1)$ \\
\hline QRS respiratory change & 33 & 56 & 17 & 5 & $86.8(71.1-95.0)$ & $76.7(65.9-85.5)$ \\
\hline P respiratory change & 30 & 41 & 32 & 8 & $73.7(56.6-86.0)$ & $56.2(44.1-67.6)$ \\
\hline
\end{tabular}

TP: True positive; TN: True negative; FP: False positive; FN: False negative; IVC: Inferior vena cava

0.63-0.82) [Figure 1e]. The best cutoff point for P wave was $\leq 12 \%$, with $73.7 \%$ sensitivity and $65.2 \%$ specificity in diagnosing CVP drop [Table 2].

\section{Designing PCQP scoring system in diagnosing low central vein pressure}

Results obtained are shown in Table 3. It can be seen that VPW $\leq 70 \mathrm{~mm}$, caval index $>34.7 \%$, changes QRS complex $\geq 20 \%$, and changes in $P$ wave $\leq 12$ were independent predictive factors to diagnose low CVP.

According to Table 4, research team named the rating system: "PCQP role in criteria." In this 13-point rating system (from
0 to 12$)$, the higher patient score means greater likelihood of low CVP. Based on ROC curve, the best cutoff point for this rating system was found 6 , which means that score $\geq 6$ is able to detect low CVP (CVP $<8 \mathrm{~cm}$ of water), with $97.4 \%$ sensitivity (95\%, CI: 84.57-99.99) and $83.6 \%$ specificity ( $95 \%$, CI: 72.65-90.86) [Figure 2].

\section{Discussion}

The results of present study showed that VPW, inferior vena cava diameter, respiratory changes in inferior vena cava diameter, QRS complex changes, and $\mathrm{P}$ wave all have a significant relationship with CVP. Accordingly, an increase in VPW, inferior 
Table 3: Multivariate logistic regression analysis results for determining the best diagnostic parameters of central vein pressure

\begin{tabular}{lcccc}
\hline Variable & $\boldsymbol{\beta}$ & $\mathbf{9 5 \%}$ Cl & $\boldsymbol{P}$ & Weight \\
\hline Over 60 years old & 0.82 & $-1.1-2.8$ & 0.41 & - \\
VPW $\geq 70 \mathrm{~mm}$ & 4.2 & $2.2-2.2$ & $<0.001$ & 4 \\
IVC diameter $\geq 10.2 \mathrm{~mm}$ & 0.7 & $-1.7-3.2$ & 0.58 & - \\
Caval index $\leq 34.7$ & 2.8 & $0.9-4.7$ & 0.004 & 3 \\
Heart/chest ratio above $50 \%$ & 1.7 & $-1.6-5.0$ & 0.31 & - \\
Changes in amplitude of QRS $\leq 20 \%$ & 2.9 & $1.0-4.7$ & 0.003 & 3 \\
Changes in amplitude of P wave $\geq 12 \%$ & 2.2 & $0.3-4.0$ & 0.03 & 2 \\
\hline VPW: Vascular pedicle width; CI: Confidence interval; IVC: Inferior vena
\end{tabular}

\section{Table 4: PCQP role in criteria rating system}

\begin{tabular}{lc}
\hline Variable & Weight \\
\hline VPW $\geq 70 \mathrm{~mm}$ & 4 \\
Caval index $\leq 34.7$ & 3 \\
Changes in amplitude of QRS complex $\leq 20 \%$ & 3 \\
Changes in amplitude of P wave $\geq 12 \%$ & 2 \\
Overall score & 12 \\
\hline
\end{tabular}

VPW: Vascular pedicle width

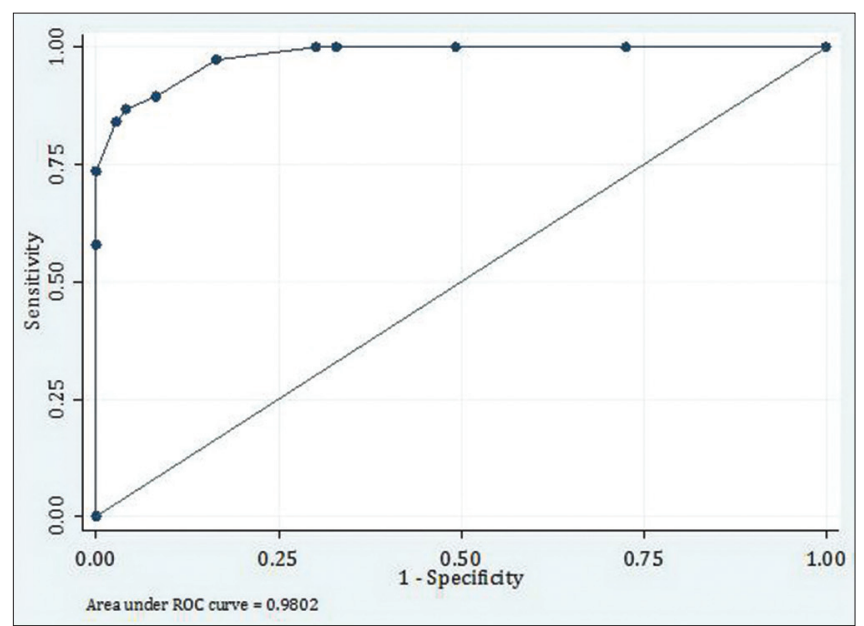

Figure 2: PCQP role in criteria receiver operating characteristic curve for detecting low central venous pressure at different cutoff points

vena cava diameter, and $\mathrm{P}$ wave amplitude change is associated with an increase in CVP, while an increase in respiratory changes in inferior vena cava diameter, and QRS complex changes is associated with a decrease in CVP. In the present study, multivariate logistic regression model was used in design of a rating system based on ultrasound and radiograph results in detecting CVP. This analysis showed greater importance of respiratory changes as compared to absolute diameter of inferior vena cava in detecting CVP. Accordingly, the researchers designed a rating model called PCQP role in criteria. According to this 13-point scoring system (from 0 to 12), lower scores show lesser possibility of CVP $<8 \mathrm{~cm}$ of water, and higher scores indicate greater likelihood of this complication.
Assessment of volume status in critically ill patients in ED ranges from simple clinical assessment and routine bedside monitoring to bedside echocardiography and different invasive/ less invasive techniques. ${ }^{[17]}$ Previous studies show that VPW is a reliable factor for assessment of circulating volume. In a review study, Ely and Haponik (2002) investigated the use of chest radiography to assess status of intravascular volume (stressing on VPW) and argued that objective assessment of VPW increases diagnostic accuracy and value of radiographic and clinical evaluations by up to $30 \%$ and even more when used in series in a patient. ${ }^{[7]}$ According to their study, the cutoff point of $70 \mathrm{~mm}$ is considered in distinguishing high intravascular volume from normal or low volume. They also suggested that a standard algorithm can be designed for VPW, and its measurement can be added to educational curriculum of physicians and radiologists. ${ }^{[7]}$ Ely et al. also argued that using VPW cutoff points above $70 \mathrm{~mm}$ and cardiothoracic ratio above 0.55 can improve assessment of intravascular volume in chest radiography. ${ }^{[8]}$

In addition to identifying low circulating volume and low CVP, VPW is also a suitable tool for monitoring positive fluid balance. For instance, Salahuddin et al. demonstrated that VPW is closely associated with positive fluid balance $(r=0.88)$ and stated that VPW $>86.5 \mathrm{~mm}$, with $100 \%$ sensitivity and $80 \%$ specificity, is indicative of body fluid volume $\geq 1200 \mathrm{~mm}$. They further stated that VPW can be considered as a reliable criterion in assessment of fluid volume in critically ill patients. ${ }^{[6]}$ Thomason et al. showed that with VPW $>63$ together with cardiothoracic ratio $>0.55$, hydrostatic pulmonary edema is more likely than edema induced by increased vascular pulmonary permeability ${ }^{[18]}$ Martin et al. reported that changes in fluid balance are reflected in chest radiograph, and serial assessment of chest radiographs can appropriately complement clinical data in controlling fluid balance in critically ill patients. ${ }^{[19]}$ The present study also showed similar results, and VPW was directly and significantly related to CVP.

The present study showed that in addition to chest radiographs and VPW assessment, changes in body fluid volume are also exhibited in inferior vena cava ultrasound. Increased or decreased collapsibility of inferior vena cava will help the management of critically ill patients. According to the results of other studies, combination of absolute diameter of inferior vena cava and its collapsibility during inhalation and exhalation provides a suitable estimate for volume status and may be used as an alternative to invasive procedures. This criterion has the following mechanism: negative pressure created during inhalation increases venous return to the heart and causes inferior vena cava to collapse. Venous return is reduced during exhalation, and diameter of inferior vena cava returns to its initial state. ${ }^{[16,20,21]}$

Percentage of vessels collapse is greater in hypovolemic, compared to hypervolemic state. This was found through calculation of caval index. In the present study, caval index above $34.7 \%$, with $89.47 \%$ sensitivity and $72.6 \%$ 
specificity, indicated CVP $<8 \mathrm{cmH}_{2} \mathrm{O}$. Various studies have shown that in many life-threatening circumstances, absolute diameter of inferior vena cava and caval index are relative to CVP. ${ }^{[21]}$ Sridhar et al. also argued that caval index is a quick, simple, noninvasive, and reliable tool for identifying intravascular volume status in crowded conditions such as ED. ${ }^{[22]}$ Nagdev et al. explained that caval index $\geq 50 \%$ can predict CVP $<8 \mathrm{cmH}_{2} \mathrm{O}$ with $91 \%$ sensitivity and $94 \%$ specificity, ${ }^{[21]}$ but there are exceptions, as well. For instance, hyperemia in inferior vena cava that may be seen in cardiac tamponade cannot provide a suitable criterion for assessment of intravascular volume, since patient's intravascular volume is either normovolemic or hypovolemic under such conditions, while ultrasound images of inferior vena cava show fluid volume overload. ${ }^{[1]}$ ECG assessment can also provide a noninvasive diagnostic test for assessment of intravascular volume status. Studies have shown that morphologic changes in ECG may reflect changes in intravascular volume. For instance, a study in 2012 showed that amplitude changes in ECG waves can be considered a suitable parameter in estimating intravascular volume. ${ }^{[14]}$ The present study showed that reduction in CVP is significantly related to increased changes in QRS complex. Moreover, this study for the first time showed that amplitude changes in $\mathrm{P}$ wave can be a useful parameter in diagnosis of critically ill patients with CVP drop.

Limitation of the study is being a single-center study which needs more trials with larger sample size for validation of the results. However, we did not use pulmonary artery catheter as a standard tool for hemodynamic monitoring, and we have to compare our study results with CVP findings. However, we excluded all patients in whom there was any risk factor for inaccurate CVP results; however, these results could differ from standard ones.

\section{Conclusion}

According to the results obtained, an increase in VPW, diameter of inferior vena cava, and amplitude changes in $P$ wave are associated with increased CVP, while an increase in amplitude changes of respiratory diameter of inferior vena cava and changes in QRS complex are associated with reduced CVP. Accordingly, the present study employed a 13-point (from 0 to 12) rating model termed PCQP, in which the lowest score indicates low and the highest score high probability of a CVP $<8 \mathrm{cmH} 2 \mathrm{O}$. The following items are included in this rating system: VPW $\leq 70 \mathrm{~mm}$ (4 points), caval index $>34.7 \%$ ( 3 points), changes in QRS complex $\geq 20 \%$ ( 3 points), and changes in amplitude of $\mathrm{P}$ wave $\leq 12 \%$ ( 3 points). According to this rating model, scores above 6 can detect the presence of CVP $<8 \mathrm{~cm}$ of water with $97.4 \%$ sensitivity and $83.6 \%$ specificity. Hence, ED physicians could use this score for the evaluation of cardiovascular and volume status of critically ill patients admitted to the ED and determine the correct therapeutic intervention to optimize organ function.

\section{Financial support and sponsorship} Nil.

\section{Conflicts of interest}

There are no conflicts of interest.

\section{ReFERENCES}

1. Alsous F, Khamiees M, DeGirolamo A, Amoateng-Adjepong Y, Manthous CA. Negative fluid balance predicts survival in patients with septic shock: A retrospective pilot study. Chest 2000;117:1749-54.

2. Maurer C, Wagner JY, Schmid RM, Saugel B. Assessment of volume status and fluid responsiveness in the emergency department: Asystematic approach. Med Klin Intensivmed Notfmed 2017;112:326-33.

3. Rivers EP, Coba V, Whitmill M. Early goal-directed therapy in severe sepsis and septic shock: A contemporary review of the literature. Curr Opin Anaesthesiol 2008;21:128-40.

4. Gray AJ, Goodacre S, Newby DE, Masson MA, Sampson F, Dixon S, et al. A multicentre randomised controlled trial of the use of continuous positive airway pressure and non-invasive positive pressure ventilation in the early treatment of patients presenting to the emergency department with severe acute cardiogenic pulmonary oedema: The 3CPO trial. Health Technol Assess 2009;13:1-06.

5. Di Marco F, Tresoldi S, Maggiolini S, Bozzano A, Bellani G, Pesenti A, et al. Risk factors for treatment failure in patients with severe acute cardiogenic pulmonary oedema. Anaesth Intensive Care 2008;36:351-9.

6. Salahuddin N, Aslam M, Chishti I, Siddiqui S. Determination of intravascular volume status in critically ill patients using portable chest X-rays: Measurement of the vascular pedicle width. Indian J Crit Care Med 2007;11:192-7.

7. Ely EW, Haponik EF. Using the chest radiograph to determine intravascular volume status: The role of vascular pedicle width. Chest 2002;121:942-50.

8. Ely EW, Smith AC, Chiles C, Aquino SL, Harle TS, Evans GW, et al. Radiologic determination of intravascular volume status using portable, digital chest radiography: A prospective investigation in 100 patients. Crit Care Med 2001;29:1502-12.

9. Milne EN, Pistolesi M, Miniati M, Giuntini C. The vascular pedicle of the heart and the vena azygos. Part I: The normal subject. Radiology 1984;152:1-8.

10. Wang H, Shi R, Mahler S, Gaspard J, Gorchynski J, D'Etienne J, et al. Vascular pedicle width on chest radiograph as a measure of volume overload: Meta-analysis. West J Emerg Med 2011;12:426-32.

11. Goldflam K, Saul T, Lewiss R. Focus On: Inferior Vena Cava Ultrasound. Vol. 6. New York: ACEP News; 2011. p. 24-5.

12. Joels CS, Sing RF, Heniford BT. Complications of inferior vena cava filters. Am Surg 2003;69:654-9.

13. Vergara GR, Wallace WF, Bennett KR. Spontaneous migration of an inferior vena cava filter resulting in cardiac tamponade and percutaneous filter retrieval. Catheter Cardiovasc Interv 2007;69:300-2.

14. Giraud R, Siegenthaler N, Morel DR, Romand JA, Brochard L, Bendjelid $\mathrm{K}$, et al. Respiratory change in ECG-wave amplitude is a reliable parameter to estimate intravascular volume status. J Clin Monit Comput 2013;27:107-11.

15. Murphy EH, Arko FR, Trimmer CK, Phangureh VS, Fogarty TJ, Zarins CK, et al. Volume associated dynamic geometry and spatial orientation of the inferior vena cava. J Vasc Surg 2009;50:835-42.

16. Blehar DJ, Dickman E, Gaspari R. Identification of congestive heart failure via respiratory variation of inferior vena cava diameter. Am J Emerg Med 2009;27:71-5.

17. Laher AE, Watermeyer MJ, Buchanan SK, Dippenaar N, Simo NCT, Motara $\mathrm{F}$, et al. A review of hemodynamic monitoring techniques, methods and devices for the emergency physician. Am J Emerg Med 2017;35:1335-47.

18. Thomason JW, Ely EW, Chiles C, Ferretti G, Freimanis RI, Haponik EF, et al. Appraising pulmonary edema using supine chest roentgenograms in ventilated patients. Am J Respir Crit Care Med 1998;157:1600-8.

19. Martin GS, Ely EW, Carroll FE, Bernard GR. Findings on the portable 
chest radiograph correlate with fluid balance in critically ill patients. Chest 2002;122:2087-95.

20. Tayal V, Blaivas M. American College of Emergency Physicians: ACEP emergency ultrasound guidelines-2001. Ann Emerg Med 2009;53:470-81.

21. Nagdev AD, Merchant RC, Tirado-GonzalezA, Sisson CA, Murphy MC.
Emergency department bedside ultrasonographic measurement of the caval index for noninvasive determination of low central venous pressure. Ann Emerg Med 2010;55:290-5.

22. Sridhar H, Mangalore P, Chandrasekaran V, Manikam R. Caval aorta index and central venous pressure correlation in assessing fluid status!“Ultrasound Bridging the Gap”. ISRN Emerg Med 2012;2012:1-5. 